\title{
CANDIDATES, SUPERVISORS AND INSTITUTIONS: PUSHING POSTGRADUATE BOUNDARIES
}

\section{AN OVERVIEW}

\section{Liezel Frick, Eli Bitzer and Ruth Albertyn}

Academic boundaries are in some ways similar to national boundaries - they are set up to colonise and govern, but at the same time are constantly challenged to reaffirm their authority and meaning. The postgraduate environment has been and is still colonised and governed by a variety of boundaries: inter/national, geographical, cultural, institutional, disciplinary and paradigmatic; also those of knowledge and relationships, and many more. The contributions to this book set out to explore and challenge such boundaries as they exist within the postgraduate environment.

The work of Thomas Kuhn (1962) and others on paradigms set the scene for establishing boundaries both within and between academic disciplines in terms of research. The earlier work of Becher and Trowler (2001) on academic tribes and their territories may also be useful to explain academics' search for a scholarly identity in the higher education environment. An academic tribe provides its members with an identity and a particular frame of reference. The characteristic identity of a particular academic tribe is developed from an early age - usually already at the undergraduate level, where patterns of thought are imprinted. These 'tribal' associations are often solidified at the postgraduate level.

The current academic environment, however, demands a certain degree of boundary permeability - academic tribes and their territories can no longer claim sole propriety of knowledge systems and paradigms, as the later work of Trowler, Saunders and Bamber (2012) and their co-workers attests. Various changes in higher education have contributed to a differentiated and permeable system, including changes in the internal characteristics of higher education institutions, rapid technological changes, an emphasis on market-friendly applied research, and external pressures for vocationally oriented curricula. The debates on so-called Mode 1 knowledge (based within specific disciplinary boundaries), versus Mode 2 knowledge (which 
refers to transdisciplinary and problem-based forms of knowledge) as espoused in the work of Gibbons (1994) and Nowotny, Scott and Gibbons (2001) have further challenged the way in which knowledge boundaries are constructed in society at large and higher education in particular. There is also the need to solve complex and large-scale problems which single disciplines or studies are unable to do. As a result, traditional knowledge systems and paradigms are challenged, as the work of Max-Neef (2005), Lather (2006) and others points out. These trends have pushed postgraduate boundaries at institutional, supervisory and candidate levels as we see the emergence of a variety of postgraduate formats, models and programmes; the practice of different forms and modes of supervision; evidence of inter-/transdisciplinary postgraduate research; and inter/national research collaboration.

Academic institutions, postgraduate supervisors and candidates are pushed to define themselves anew amidst these moving boundaries. Barnett (2011) rightfully argues that universities currently function within the uneasy space between idea and reality, and that there is not a simple uniform idea of the university itself, or the way in which it produces knowledge. The complexity of the university, its surroundings and the diversity of its inhabitants demand a variety of approaches to establishing and supporting knowledge production through research. Understanding and implementing a strategy towards this end implies a delicate but functional relationship between higher education institutions, supervisors, and postgraduate candidates. This book provides a range of ideas on how the notion 'boundaries' (and the permeability thereof) influences institutions, supervisors and candidates at the postgraduate level. This book therefore focuses on the following (contested) boundary areas:

- knowledge boundaries;

- expansion and risk;

- doctoral writing;

- supervision strategies;

- supervising across cultures; and

- doctoral experiences and identities.

While these might not be the only boundaries that exist or are pushed within the postgraduate domain, they are key to how we understand (and challenge) the functioning of postgraduate systems.

Knowledge lies at the core of the postgraduate endeavour, and therefore it is apt to consider the role of knowledge boundaries within the changing postgraduate supervision landscape. Sue Clegg makes a convincing argument in her chapter 
that we need to pay greater attention to knowledge questions in doctoral education - particularly the understanding of knowledge and the need to theorise it more adequately. Bernstein's work is used as a basis to argue for the importance of theorising the differences between disciplines and for looking at the processes of regionalisation in understanding doctoral practices, as there seems to be a greater emphasis on knowledge production in the doctorate than in other areas of the curriculum. 'Knowledge questions and doctoral education' pushes knowledge boundaries in understanding doctoral education as more than simply research, but as pedagogy. This chapter does not necessarily bring new research, but it does bring new conceptual perspectives to those who research or facilitate postgraduate education. This is an important chapter for its emphasis on the role of knowledge in doctoral education and its emphasis on theorizing the role and the diversity of knowledge practices which are constitutive of doctoral education. In his chapter, Terry Evans uses contemporary research, policy and scholarship on doctoral studies, as well as the work of Kuhn and Mulkay to consider the boundary-riding and boundary-breaking work through which supervisors and candidates push knowledge boundaries. Knowledge boundaries are pushed by considering the process of undertaking a doctorate as one of working within and beyond boundaries to produce both an original contribution to knowledge (embedded in the thesis) and a new researcher in the field (the doctoral graduate). Doctoral work, therefore, works not only within a discipline's boundaries, but also within the boundaries of doctorateness.

The massification of higher education across the globe has also influenced the boundaries of postgraduate education and supervision, forcing traditional boundaries to expand. Chaya Herman's chapter focuses on the South African doctorate in the context of massive global expansion of postgraduate education which has been taking place over the last two decades. The impact this expansion has on the future of doctoral education in South Africa in terms of policy, practice and research and the role that academics and the PhD should play in knowledge creation is explored in terms of existing and expanding boundaries. Such expansion does not take place without risk. Liezel Frick, Ruth Albertyn and Eli Bitzer take up the question of risk in doctoral education with a particular focus in their chapter on how the concept of risk currently operates in doctoral education, based on a conceptualisation of risk in general and a small-scale study with experienced doctoral supervisors across disciplines at one South African university. They argue that risk is not an inevitably negative concept, but also seems to provide opportunities for increased and higher levels of scholarly performance and results. By identifying ways of containing risk, postgraduate supervisors may be better equipped to facilitate the process of student 
development towards an original contribution at a doctoral level, thereby being capable of dealing with the pressures of inevitable boundary expansion.

Doctoral writing fulfils a dual role - that of writing to know and knowing how to write (Aitchison 2010). This notion of doctoral writing forces us to ask whether (and if so, how) we support students' ability to contribute to the scholarly debate. James Burford reports in his chapter on doctoral writing as an affective practice which can be tied to the precarity of academic labour and what implications this may have for supervision. This position enables us to better account for the layering of affect within the lives of students, as well as its patterned distribution across doctoral education. It also helps us to unpack the complexities of doctoral writing and be(com)ing a doctoral writer. Pia Lamberti and Albert Wentzel provide insights into how authoritative disciplinary voices can be integrated into postgraduate writing. The disciplinary boundary crossing promoted by the collaboration between the two authors allows for a more nuanced and multifaceted understanding of the specific language and discursive resources required for the writing of the literature section of the dissertation or thesis. They also problematise the boundary between undergraduate and postgraduate level study, and suggest that research supervision necessarily encompasses a greater degree of explicit teaching than supervisors conventionally acknowledge.

Higher education institutions play a central role in determining the boundaries of postgraduate supervision. These institutions also provide support structures within these boundaries that aim to support postgraduate supervisors and that fit within the boundaries of the institution. However, new forms and modes of postgraduate programmes, supervision and research are emerging that may challenge the existing institutional boundaries. These challenges create the opportunity to explore how institutions relate and respond to such changes. At the same time supervisors play a key role as they negotiate boundaries between the different scholarly roles, engage in the formation and sustaining of communities of scholarly practice, conduct and reflect on forms of innovative supervision and review and revitalise existing supervisory practices. In this book, Callie Grant takes the position that the liberal notion of supervision with its traditional one-to-one relationship between student and supervisor can no longer be considered the only supervision strategy in the higher education arena in South Africa. Conceptualising supervision as being relational within a scholarly community of practice could offer beneficial alternatives and opportunities within the current diverse and complex society in terms of expanding and enriching the teaching and learning process. In a related chapter, Margaret Kiley, Joe Luca and Anna Cowan ask why coursework in Australian PhD programmes is a boundary and how it is being pushed - as different universities 
and disciplines have approached this supervision strategy with different concepts of coursework, its purpose, content, and placement within candidature. They examine the understandings of staff and students at two different universities on what the introduction of 'coursework' in the Australian PhD might entail - and how this might push our understanding of supervising strategies. Nonnie Botha identifies some of the ways in which cohort supervision facilitates doctoral success, by mapping Bitzer's conceptual framework for exploring doctoral success onto the outcomes of a small-scale empirical study and seeking confirmation of such indications in South African and international literature. She offers some suggestions for improving the modalities of the cohort supervision model, adding to the body of knowledge on cohort supervision as supervision strategy and thus pushing the boundaries in postgraduate supervision. In the final chapter of this section, Khalid El Gaidi uses a journeyman analogy of doctoral studies to both challenge and help us understand how the nature and communication of skills result in the development of knowledge - particularly in complex areas such as music and acquiring a native or specialised language. Due to increased numbers of candidates per supervisor, students have less time to spend with their supervisors for adequate skills transfer. Through working together with more proficient peers in research and reflecting on their experiences (such as in the journeyman's experience) PhD students are able to acquire a sense of quality in research in writing, experimenting and review. Using dialogue seminars and a pragmatic approach based on the language philosophy of Wittgenstein, Khalid El Gaidi suggests a knowledge taxonomy to analyse the specifics of skills and proposes experience-based learning to acquire such skills.

Contemporary society presents postgraduate supervisors with a diverse student body, which often demands that institutional, relational, individual and knowledge boundaries be reconsidered. Universities today are marked by increasing staff and student mobility, flexibility in programmes, multinational research partnerships, joint international appointments and publication and, most importantly, free exchange of knowledge. In order to maximise the benefits of internationalisation and ensure sustained collaboration, high levels of intercultural competence are required from all parties. Institutional, supervisory and candidate reactions to such boundary challenges may differ, therefore Michelle Picard, Cally Guerin and lan Green propose to push the boundaries of research education through a coordinated framework for developing researchers' intercultural competency. Puleng Motshoane and Sioux McKenna focus on the integral roles that structure and culture have to play in postgraduate supervision, alongside the well-documented roles played by the supervisors themselves. They challenge policy makers and institutional management to consider 
the ways in which structural and cultural issues affect postgraduate supervision and how these can be fostered to improve the postgraduate supervisory process.

Postgraduate students themselves may be instrumental in pushing boundaries. Their lived experiences of supervisory practices or how they engage with boundaries as part of their postgraduate experience are valuable contributions to understanding how boundaries contribute to or hinder their ultimate success. These boundaries may relate to personal (motives for doing research, preferred learning style, confidence, past experiences, ideological perspective), social (cultural background and gender) or geographical (international students) issues. Catherine Mitchell reflects on the role of supervisors supporting first-generation students who aspire to live the academic dream. She provides a reflective account of the multifaceted nature of the supervision process from her perspective as a student. Her contribution helps us to consider what students learn from their supervisors as they imagine an academic future and how supervisors may contribute to students' movement into academia. In the penultimate chapter, Susan van Schalkwyk argues that doctoral studies represent 'complex investments' for those who embark on them. Drawing on data generated among a number of doctoral candidates who meet on a monthly basis, she sought to understand the nature of these complex investments. For such understanding she uses the work of Margaret Archer, who describes identity formation occurring through the personification of a particular role and requiring an investment or intentionality on the part of the doctoral candidate. Van Schalkwyk suggests that if supervisors hope to foster the development of a doctoral identity, then there may be a need to understand what mediates candidates' internal conversations. Such understanding may better enable them to establish nurturing spaces towards identity formation. This may require that the shroud obscuring the inner workings of the supervisory relationship is disturbed and in so doing, that the boundaries that currently define it are challenged.

\section{REFERENCES}

Aitchison C. 2010. Learning together to publish. In: C Aitchison, B Kamler \& A Lee (eds). Publishing pedagogies for the doctorate and beyond. Oxon: Routledge. 83-100.

Barnett R. 2011 . The coming of the ecological university. Oxford Review of Education, 37(4): 439-455.

Becher T \& Trowler P. 2001. Academic tribes and their territories: Intellectual inquiry and the culture of disciplines. Buckingham: Society for Research into Higher Education.

Gibbons M. 1994. The new production of knowledge: The dynamics of science and research in contemporary societies. London: Sage.

Kuhn T. 1962. The structure of scientific revolutions. Chicago, IL: University of Chicago Press. 


\section{CHAPTER 1 • CANDIDATES, SUPERVISORS AND INSTITUTIONS: PUSHING POSTGRADUATE BOUNDARIES}

Lather P. 2006. Paradigm proliferation as a good thing to think with: Teaching research in education as a wild profusion. International Journal of Qualitative Studies in Education, 19(1):35-57.

Max-Neef MA. 2005. Foundations of trandisciplinarity. Ecological Economics, 53:5-16.

Nowotny H, Scott P \& Gibbons M. 2001. Re-thinking science: Knowledge and the public in an age of uncertainty. Cambridge: Polity.

Trowler P, Saunders M \& Bamber V (eds). 2012. Tribes and territories in the $21^{\text {st }}$ century: Rethinking the significance of disciplines in higher education. Florence: Routledge. 